\title{
Praxeologische Feldforschung - Reichweite, Tragweite, Importanz und Relevanz als Analysekategorien
}

\author{
Klaus Geiselhart ${ }^{1}$, Simon Runkel ${ }^{2}$, Susann Schäfer ${ }^{2}$, and Benedikt Schmid ${ }^{3}$ \\ ${ }^{1}$ Institut für Geographie, Friedrich-Alexander Universität Erlangen-Nürnberg, 91058 Erlangen, Deutschland \\ ${ }^{2}$ Institut für Geographie, Friedrich-Schiller Universität Jena, 07743 Jena, Deutschland \\ ${ }^{3}$ Institut für Umweltsozialwissenschaften und Geographie, Albert-Ludwigs-Universität Freiburg, \\ 79098 Freiburg, Deutschland \\ Correspondence: Simon Runkel (simon.runkel@uni-jena.de)
}

Received: 15 April 2020 - Revised: 11 January 2021 - Accepted: 25 January 2021 - Published: 12 March 2021

Kurzfassung. This paper develops three analytical categories - range, supporting capacity, exigency/notability - to capture how supra-individual phenomena affect the people studied by empirical research. Researchers face a tension between constructivist and realist perspectives as the examined phenomena are simultaneously social constructs, in the way people perceive and understand them, and social facts in their consequences. Taking a critical perspective on the notion of large social phenomena - popularized by Theodore Schatzki - the paper develops an explorative terminology that aims to facilitate practice-oriented field research. Examples of empirical research on transition and degrowth initiatives illustrate how research subjects estimate the range of a phenomenon by trying to grasp whether they are in or out of its reach; the supportive capacity of a phenomenon by exploring how far it carries certain processes; and they experience the exigency of a phenomenon and ascribe a certain notability to it. Taken together, this terminology grasps the way phenomena are matters of concern, rather than matters of fact, for the research subjects.

\section{Einleitung: Empirisches Arbeiten zwischen Realismus und Konstruktivismus}

Empirische Forschungspraxis spielt für Humangeograph*innen eine elementare Rolle beim Verstehen und Analysieren sozialräumlicher Sachverhalte. Wenn Forschende ins Feld gehen, erkennen sie häufig - wie wir es bei eigener Feldforschung erfahren haben -, dass die Beforschten bestimmte Dinge als gegebene Tatsachen ansehen, die in akademischen Diskursen als sozial konstruiert und kontingent identifiziert werden. In Interviews mit Beforschten wird auf verschiedene Phänomene Bezug genommen, die diese als Bestandteile ihrer Wirklichkeit ansehen. Dieser Blick bestimmt schließlich auch die Handlungsdispositionen der Beforschten und entsprechend die sozialen Verhältnisse, die Wissenschaftler*innen analysieren wollen und die aus wissenschaftlicher Perspektive vielleicht auch kritisierbar erscheinen. Aus einer praxeologischen Perspektive erkennen Forschende einen Kreislauf von Konstruktion, entsprechenden Handlungen und erneuter Reproduktion der Verhältnisse.

Mit dieser Erkenntnis geraten Forschende aber in ein Dilemma. Sie müssen erst einmal die Setzungen der Beforschten auf die ein oder andere Weise akzeptieren. Nicht allein, weil das empirische Interesse eine Anhörung spezifischer Sichtweisen, wenn nicht gar deren Reflexion verlangt, sondern auch schon, weil Forschende ihr Untersuchungsfeld und die Beforschten beschreiben müssen. Sie dürfen also die Existenz des Phänomens nicht initial in Frage stellen. Dabei können sie sich natürlich der Gemachtheit von sozialen Sachverhalten bewusst sein. Erklären sie aber die Konstruktion des besonderen Phänomens, indem sie lediglich dessen Reproduktion beschreiben, dann reifizieren sie damit das Phänomen. Damit akzeptieren Forschende stillschweigend auch dessen Status als existenten Sachverhalt. Damit nehmen sie letztlich zwar eine kritische, aber doch realistische Perspektive ein. 
Deutlich wird dies beispielsweise bei einem Sachverhalt, der trotz umfassender Kritik häufig als gegeben angesehen wird: der Markt. Die kritischen Wissenschaften lehnen die neoklassische Annahme eines abstrakten, unabhängigen und ausgleichenden Marktes ab (Gibson-Graham, 1996, 2006; Massey, 2012). Im allgemeinen Verständnis wird Markt jedoch meist weiterhin als Faktum vorausgesetzt - nicht zuletzt in akademischen Betrachtungen selbst (Berndt und Boeckler, 2009). Ansätze der Diskurs- und Performativitätsforschung zeigen indes, dass Konzepte bestimmend Einfluss auf Praktiken und Prozesse nehmen und damit wiederum soziale Phänomene wie das des Marktes bedingen (MacKenzie et al., 2008; Cohen, 2017; Aspers, 2007; Ouma und Bläser 2015). Schauen Forschende aber darauf, wie ein bestimmter Sachverhalt durch Mitwirken seiner Teilnehmer*innen performativ hergestellt wird, so nehmen sie letztlich eine sozialkonstruktivistische Perspektive ein. Im Mittelpunkt steht die Hervorbringung eines Sachverhaltes, aber dennoch bleibt der Sachverhalt ein ,matters of fact". Diesen Sachverhalt können Forschende zwar rekonstruktiv, also in seiner sozialen Hervorbringung, beschreiben, doch wird er in deren Beschreibung zwangsläufig verdinglicht.

Erfahrungen von ,,im Feld“ Forschenden haben aber gezeigt, dass die Beforschten eigene individuelle Haltungen zu gängigen, konventionalisierten Vorstellungen eines sie betreffenden Sachverhaltes haben. Vielleicht haben Forschende auch gesehen, wie Beforschte versuchen auf ihre ganz eigene Art und Weise ihre individuellen Vorstellungen in Wert zu setzen, um - im Beispiel zu bleiben - ökonomisch tätig zu sein. Letztlich sehen Forschende sich in dem Dilemma, zwischen einem Blick auf die soziale Konstruktion eines Phänomens und einem Blick auf die Realität seiner Existenz entscheiden zu müssen. Empirisch Forschende können nun versuchen, zwischen einem konstruktivistischen und einem realistischen Blick zu alternieren, was aber mit erheblichen methodischen Schwierigkeiten und Problemen bei der Formulierung der Ergebnisse einhergeht.

Praxeologische Ansätze nehmen für sich in Anspruch, Dichotomien in Frage zu stellen, und wir wollen im Folgenden untersuchen, ob sie nicht auch eine Lösung für das hier beschriebene Problem bereithalten. Mit dem Begriff der Praxis lässt sich der Dualismus von individueller Aktivität und sozialer Struktur überwinden, indem man nun ,,überindividuelle [...],Muster ' im fortlaufenden Tätig-Sein von Individuen“ (Geiselhart et al., 2019, 27) identifiziert, welche von Individuen mehr oder weniger automatisiert ausgeführt werden. Dabei ist von großer Bedeutung, wie diese Muster verstanden werden. Werden sie ontologisiert, also als kleinste identifizierbare Einheiten des Sozialen angesehen, die als ganz spezifische Muster existieren, oder werden sie als Rahmungen von Vielfalt angesehen? Im ersten Fall sind sie den Subjekten vorgängig, werden von diesen lediglich ausgeführt, und Veränderungen in den Praktiken ergeben sich nur zufällig durch Iteration. Im zweiten Fall ermöglichen Praktiken den Subjekten einen gewissen Spielraum, innerhalb dessen sie in einem gewissen Maße individuell handeln können oder sogar eine gewisse agency besitzen.

Noch deutlicher wird diese Spannung, wenn man sich größeren Zusammenhängen des Sozialen zuwendet. Mit seiner Konzeption der ,large social phenomena“ vertritt Theodore Schatzki (2016) eine praktikenontologische Perspektive. Er hat sich sehr konkret zu der Herausforderung praxeologischer Forschung geäußert, größere Zusammenhänge menschlichen Tätig-Seins konzeptionell zu fassen. Wie im Folgenden zu zeigen ist, birgt diese in der humangeographischen Forschung zunehmend einflussreiche Konzeption Schatzkis jedoch einige Probleme. Schatzki definiert „large social phenomena" als ,spatially extensive, consisting in a far-flung constellation of practices or arrangements “ (Schatzki, 2016, 6). Dadurch versucht Schatzki, soziale Phänomene - wie beispielsweise Märkte - als komplexes Ineinandergreifen einer großen Zahl von Tätigkeitsmustern und materiellen Arrangements zu verstehen. Schatzkis Konzept verbleibt dadurch in einer ontologisierenden Deskription des Seins eines Sachverhaltes und eröffnet damit keinen Zugriff auf die Wirkmächtigkeit sozialer Sachverhalte oder Phänomene. Damit gelingt es nicht, den methodologischen Widerspruch zwischen Realismus und Konstruktivismus zu überwinden. Phänomene sind, so argumentieren wir im Folgenden, beides zugleich: konstruiert, wie auch real. Sie sind konstruiert in ihrer Wesenhaftigkeit und real in ihren Auswirkungen. Als Alternative werden wir mit Verweis auf Bruno Latours Unterscheidung zwischen ,,matters of fact“ und ,,matters of con$c e r n$ " eine Analytik zur empirischen, praxeologischen Erforschung von überindividuellen Sachverhalten anbieten.

Dieser Beitrag gliedert sich wie folgt. Zunächst werden wir in aller Kürze theoretische Ansätze zu sozialen Praktiken umreißen (Kapitel 2.1). In einem nächsten Schritt werden wir darauf aufbauend Schatzkis Begriff der ,large social phenomena" kritisch diskutieren, um ihn dann beispielhaft auf das Phänomen Markt zu beziehen (Kapitel 2.2). Wir werden zeigen, wie in Schatzkis Konzept eine Gefahr der Reifikation angelegt ist. Schließlich skizzieren wir im dritten Kapitel erste methodologische Überlegungen, wie mittels der Analysekategorien Reichweite, Tragweite, Importanz und Relevanz ein alternativer Zugang zur Betrachtung überindividueller Sachverhalte gelingen kann. Die Analysekategorien entwickeln wir entlang empirischer Beispiele aus alternativwirtschaftlichen Projekten, die versuchen, sich marktförmigen Produktions- und Distributionsverhältnissen zu entziehen und somit die Grenzräume von ,Märkten“ sichtbar machen. Die anonymisierten Beispiele sind einem in seiner Methodik praxistheoretisch ausgerichteten Forschungsprojekt entnommen (Schmid, 2020). Sie dienen den methodologischen Überlegungen in diesem Beitrag lediglich zur Illustration. ${ }^{1}$ Abschließend sammeln wir in einer Konklusi-

\footnotetext{
${ }^{1}$ Nebst Märkten lässt sich die hier vorgeschlagene Kritik und Analytik auf weitere Sachverhalte beziehen wie beispielsweise auf so unterschiedliche Frage- und Problemstellungen wie die der ge-
} 
on (Kapitel 4) ausblickhaft verschiedene Überlegungen für zukünftige empirische Untersuchungen und theoretische Anschlussmöglichkeiten.

\section{Theoretische Rahmung}

\subsection{Theoretische Hintergründe sozialer Praktiken}

Praxeologische Perspektiven haben in den letzten Jahren große Resonanz erfahren, sodass Schatzki, Knorr-Cetina und Savigny von einem ,practice turn“ (2001) sprechen. Die Rede von einem ,turn“ überdeckt allerdings die Tatsache, dass in den vorausgegangenen Jahrzehnten sehr prominent und einflussreich praxeologische Theoriebildung betrieben wurde, die insbesondere mit Namen wie Bourdieu, Dreyfus, Giddens, Latour, Laclau und Mouffe, Taylor - und eben auch Schatzki - verbunden ist (für eine Übersicht siehe Geiselhart et al., 2019).

Im Kern geht es bei praxeologischen Perspektiven darum, Strukturen und Akteure - klassische Bezugspunkte unterschiedlicher Sozialtheorien - nicht als etwas Erklärendes vorauszusetzen, sondern diese selbst als soziale Vollzugsformen zu verstehen. Praxeologien, in anderen Worten, legen nicht im Vorhinein fest, ,aus welchen Struktureigenschaften oder Handlungsintentionen die Sozialität emergiert“ (Hillebrandt 2014, 11). Stattdessen, gehen sie davon aus, dass soziale Phänomene wie Märkte oder der Staat selbst Produkte fortwährenden menschlichen Tätig-seins sind (sozial konstruiert), gleichzeitig aber auch auf dieses Tätig-sein einwirken (real in ihren Auswirkungen).

Praxistheorien zielen somit darauf ab, zum einen eine Alternative zum ontologischen Primat einer gesellschaftlichen Totalität, wie zum Beispiel in der Systemtheorie oder im Funktionalismus zu bieten, und zum anderen auch dem Individuum keine sozialontologische Vorrangstellung einzuräumen wie etwa in der neoklassischen Ökonomik, dem methodologischen Individualismus (Werlen, 1999, 33) oder dem symbolischen Interaktionismus (Schatzki, 1996, 9). Praxistheoretische Ansätze verfolgen ein grundlegend prozessuales Verständnis von Wirklichkeit. Soziale Phänomene werden in ihrer geschichtlichen Bedingtheit und Genese verstanden und damit als kontingenter, gleichzeitig jedoch materieller Vollzug. Ziel eines praxeologischen Blicks ist es daher nicht, Praktiken zu fixieren, das heißt sie ontologisch als Entität zu etablieren. Epistemologisch lassen sich praxeologische Ansätze folglich als (Versuch einer) Überwindung des Gegensatzes zwischen konstruktivistischen und essentialistischen Positionen fassen. Stattdessen wird ein Konzept von

sellschaftlichen Integration von sozialen Gruppen, der Stadtplanung, der Klimawandelanpassung oder der kritischen Geopolitik. In religionssoziologischen und -geographischen Untersuchungen tritt das Problem zudem häufig unter dem Stichwort ,,methodologischer Agnostizismus“ (vgl. Henkel, 2011) auf, wenn Feldforschende mit der von den religiösen Menschen für Realität gehaltenen Glaubensüberzeugungen konfrontiert werden.
Praktiken vorgeschlagen, womit der grundlegend prozessuale Charakter gesellschaftlicher Wirklichkeit zu fassen ist.

\subsection{Schatzkis Praktikenontologie als Vergleichsfolie}

Ein wichtiger Name der jüngeren praxeologischen Theoriebildung ist Theodore Schatzki. Er erarbeitete eine spezifische Denkweise, die sich als praktikenontologisch bezeichnen lässt, die uns als Vergleichsfolie dienen soll. Schatzki (2003, 2008, 2010) versteht Praktiken als Bündel von Aktivitäten, im Tun und Sagen (,,doings and sayings“) durch praktisches Verstehen (,practical understandings"), Regeln bzw. Vorschriften (,,rules"), teleo-affektive Strukturen, d. h. leibliche und geistige Ausrichtungen (,teleo-affective structures"), sowie generelles Verstehen (,general understandings"). Für Schatzki stellen Praktiken den zentralen Aspekt des sozialen Lebens dar. In ihrer Verbundenheit konstituieren sie das Feld des Sozialen (Schatzki, 1996, 198 ff.). In jüngeren Arbeiten ergänzt er dies mit einer (flachen) Ontologie der ,site“ (Schatzki, 2002, 123 ff.): ,[...] something occurs in a site when it is inherently a part of a context in which it occurs. Site ontologies either locate the social in or identify it with some site" (Schatzki, 2002, 138). Damit bezieht seine Praxistheorie materielle und immaterielle Entitäten mit ein. Dies wird durch eine weitere wesentliche Konzeption in seiner Praxistheorie ergänzt: soziale Ordnungen und Arrangements. Soziale Ordnungen nennt er Ensembles von Entitäten, durch welche sich soziales Leben dynamisch entfaltet (Schatzki, 2002, 38). Gemeint sind damit in seinem Vokabular Arrangements von Menschen, Artefakten, Organismen und Dingen, die für menschliche Koexistenz charakteristisch sind (ebd.). In solchen Ordnungen verbinden sich Entitäten, teilen Bedeutung und sortieren sich in gegenseitiger Anerkennung (ebd.). Die sozialen Ordnungen schließen dabei materielle und immaterielle Arrangements mit ein. Arrangements bezeichnen die gegenseitige Lage oder Position von Entitäten zueinander (vgl. Löw, 2001), wobei Schatzki $(2002,19)$ ausdrücklich darauf hinweist, dass diese Position nicht notwendigerweise ein räumliches Phänomen sein muss. Vielmehr sei „Position“ ein ,,abstract term denoting where an entity fits in a nexus" (ebd.). Schatzkis - zuweilen sehr komplexes und nominalistisches - Vokabular ist für humangeographische Perspektiven attraktiv und wurde in diesem Kontext bisher nicht nur konzeptionell aufgearbeitet (Everts et al., 2011; Schäfer und Everts, 2019), sondern bereits in verschiedenen empirischen Untersuchungen operationalisiert (Lahr-Kurten, 2014; Haubrich, 2015; Maus, 2015).

Schatzkis Theorie hat ihre Stärke darin, dass sie das Soziale in Begriffe zerlegt, die sich gut operationalisieren lassen. Gleichzeitig - so gesehen eine Schwäche des Ansatzes besteht dadurch eine Tendenz zum reifikatorischen Umgang mit diesen Begriffen. Während andere praxeologische Ansätze Praktiken eher in deren Effekten und Wirkungen (z. B. Disziplinierungen des Selbst) und diese im Hinblick auf Gesellschaft, Klasse und/oder soziale Gruppe analysieren, be- 
trachtet Schatzki ,soziale Praktiken” als kleinste Einheit des Sozialen, woraus sich vielfach der Impuls der empirischen Identifizierung speist. Das lohnenswerte an Schatzkis Vorgehen ist, dass er soziale Praktiken damit als empirische Analysekategorien handhabbar macht und auch Hinweise auf deren Einbindung in größere Arrangements bietet.

In seinen jüngeren Arbeiten setzt sich Schatzki mit der Frage auseinander, wie man mit seiner Theorie größere $\mathrm{Zu}$ sammenhänge des Sozialen denken kann. Er entwickelt den Begriff der ,large social phenomena“ (Schatzki, 2016). Nach eigenen Aussagen reagiert Schatzki damit auf die Kritik, man könne sich mit seiner Praxistheorie nur Mikrophänomenen zuwenden. „Some theorists say that practice theory applies best - or even only - to small social phenomena" (Schatzki, 2016, 4). Schatzki führt argumentativ Bourdieus soziale Klassen an. Diese beispielsweise seien große Phänomene, denn sie durchdringen und vermischen verschiedene von Bourdieu beschriebene Felder (ebd., 5). Es fehle aber, so seine Auffassung, ein Begriff davon, was große soziale Phänomene allgemein seien. Einen solchen Begriff entwickelt Schatzki:

„A social phenomenon is anything that pertains to human coexistence. Because social life transpires as part of the plenum of practices, social phenomena consist in slices, sectors, or sets of features of this plenum (of the bundles and constellations that compose it). Social phenomena differ in the continuity, density, and spatial-temporal form of the practices, arrangements, bundles, and relations among practices, arrangements, and bundles that compose them. Two important features of this plenum, and thus of social phenomena, are density and size (others are duration, shape, and qualitative complexity). Density is the number of activities, entities, relations, practices, bundles, and constellations per unit of three dimensional space. As noted, greater relational density marks the existence of bundles and constellations. Size, meanwhile, is the small-large spectrum of spatial extension. A ,large' social phenomenon is one that is spatially extensive, consisting in a far-flung constellation of practices or arrangements" (Schatzki, 2001, 6)

Schatzki ist der Ansicht, soziale Phänomene seien Ausschnitte, Sektoren oder Kombinationen von Bestandteilen eines größeren Plenums der Gesamtheit aller PraktikenArrangement-Bündel. Sie hätten eine Dichte, Größe, Dauer, Form und eine qualitative Komplexität bezüglich ihrer Konstellationen. Das sind alles Beschreibungen, die soziale Phänomene verdinglichen und implizit dazu auffordern, sie empirisch in diesen Eigenschaften (Dichte, Größe, Form und Komplexität) abzuschätzen. „Large social phenomena“ werden damit explizit nach deren Größe bemessen, die ganz materiell als räumliche Ausdehnung verstanden wird, genauso wie Dichte als Grad der Durchdringung des euklidischen
Raumes verstanden wird. Damit hebt diese Definition darauf ab, soziale Phänomene als Entitäten im praxeologischen Denken zu etablieren. Wie wir im Folgenden zeigen, birgt Schatzkis nominalistische Definition von ,large social phenomena" jedoch die Gefahr, dass Forschende, die sich an diesem Konzept orientieren, in der Empirie die Dynamik und Multiplizität der Praxis aus dem Blick verlieren.

\subsection{Kritik an Schatzkis Konzept der „Large social phenomena"}

Dass Schatzki sein Denken als grundsätzlich ontologisches Unterfangen ansieht, mag vor dem Hintergrund des Wahrheitsrelativismus des ,cultural turn“ befremdlich anmuten, doch tatsächlich ist sein Nominalismus, wie schon erwähnt, insofern zuträglich, als dass er Kategorien vorschlägt, die in der empirischen Forschung der Orientierung dienlich sein können. Im Folgenden argumentieren wir aber, dass dieses ontologische Unterfangen Schatzkis an Schlagkraft verliert, wenn er sich mit dem Begriff der ,large social phenome$n a$ “, wie er schreibt, ,größeren“ Zusammenhängen des Gesellschaftlichen zuwendet.

Zunächst ist fraglich, ob es überhaupt eines spezifischen praxeologischen Begriffs großer Phänomene bedarf. Unseres Erachtens nach vermögen es praxeologische Ansätze durchaus, sich bedeutenden Dingen bzw. großen Sachen (,, big issues") zuzuwenden, so etwa Giddens mit dem Machtbegriff der Ressourcenverfügbarkeit, Bourdieu mit den Lebensstilen oder Foucault mit Praktiken der Disziplinierung des Selbst: Damit beschreiben diese Autoren bedeutende Phänomene, die überindividuell starken gesellschaftlichen Einfluss ausüben. Auch sind Praktiken nicht per se „klein“. Die Praktik des Verkaufens beispielsweise steht in Zusammenhängen, die weit über das unmittelbare Umfeld ihrer jeweiligen Ausführungen hinausreichen. Die Stärke von Praxistheorien liegt gerade darin, empirisch die tatsächliche Institutionalisierung einer Praktik, mittels Beobachtung konkreter, praktischer, singulärer Ereignisse, die mit dieser Praktik verbunden sind oder durch die sie hervorgebrachten werden, zu beschreiben.

Schatzki versucht sich jedoch an einer Ontologie von, ,lar-

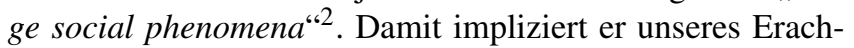
tens auch eine Suche nach derartigen Phänomenen. In der Empirie birgt das die Gefahr, dass die Praktik (bspw. die des Verkaufens) aus dem Blickfeld gerät und die Tendenz entsteht, das aus ihr resultierende Phänomen (den Markt) zu beschreiben. Dabei entsteht die Gefahr, dass diese Phänomene

\footnotetext{
${ }^{2}$ Von einer Übersetzung von ,large social phenomena“ in große soziale Phänomene sehen wir insofern ab, als dass ,large“ nur unzureichend mit ,groß“ übersetzt werden kann. ,Large“ kann ebenfalls mit umfangreich, weitreichend und reichlich übersetzt werden, sodass in diesem Ausdruck in gewisser Hinsicht auch eine (räumliche) Ausdehnung mitschwingt. Dieser Konnotation werden wir im Fortgang der Argumentation mit den Begriffen Trag- und Reichweite Rechnung tragen.
} 
(Märkte) von ihrer performativen Herstellung abgekoppelt werden, um sie als ein großes Prinzip (der Markt) zu reifizieren. Eine praxeologische Analyse müsste aber stattdessen danach fragen, ob die Konventionen der Praktiken im lokalen Kontext so zwingend sind, als dass nicht einzelne Individuen darin auch Nischen und/oder individuelle Verfahrensweisen entdecken.

Schatzki (2001) hingegen fordert dazu auf, von ,large social phenomena" auszugehen und diese kausal zu erklären. Denn ,the complexity of the action chain nexuses involved requires the provision of overviews" (ebd., 22). Auch wenn Schatzki hier keine strenge Form von Kausalität zwingender, ausschließlicher Wirkungen denkt, sondern unter Kausalität lediglich eine wie auch immer geartete Einflussnahme versteht, so erinnert das doch an rationalistische oder intellektualistische Bestrebungen, Gesellschaft abzubilden und zu erklären.

Hervorzuheben ist auch, dass Schatzki verschiedenste Kategorien aus ganz unterschiedlichen Theoriedebatten kombiniert, die die Myriaden von kleineren Veränderungen beschreiben sollen, die in ihrer Komplexität die Dynamik des Wandels eines "large social phenomena“ letztlich ausmachen. Die Zusammenhänge von Handlungsketten (,,action chain nexus") nehmen Gestalt an durch: ,feedback“, ,domestication“, „,cascades“, „,bifurcations“, ,governance“, „,monitoring “, ,coordinating devices", ,coordinating discourses" und „material infrastructures“. Schatzki (ebd., 18) erläutert: „Such concepts are needed for giving fuller causal explanations of the formation, perpetuation, and dissolution of large phenomena“. Auch wenn sein Hauptargument komplexitätstheoretischer Natur ist, so bezieht Schatzki mit Diskursen, Artefakten und Governance auch Konzepte aus anderen Theoriedebatten mit ein. Auf der einen Seite ist das nachvollziehbar, da in komplexen Systemen tatsächlich multikausale Einflüsse stattfinden und auch intentionale Strategien und handlungstheoretisch beschreibbare Prozesse durchaus denkbar sind. Dabei aber scheint Schatzki zu verkennen, dass die Feststellung derartiger Kausalitäten auf der Ereignisebene es nicht erlauben, Emergenzen auf der Phänomen-Ebene zu erklären. Schatzki behauptet aber, dass so beispielsweise die Emergenz eines Netzwerks an Global Cities als Rückgrat der globalen Finanzmärkte verstanden werden kann (ebd., 22).

Zusammenfassend bleibt eine Terminologie, die ausgehend von ,large social phenomena" deren Wandel mittels einer Reihe von Begrifflichkeiten zu ergründen sucht. Dies aber entspricht dem, was Schatzki selbst als skalares Denken kritisiert. Der Analyse sollten keine vorab definierten hierarchisch geordneten Kategorien, von Mikro zu Makro oder lokal bis global, bestimmte Charakteristika zu Grunde gelegt werden (Marston et al., 2005). Demnach bedarf es einer flachen Ontologie, um die Effekte der Naturalisierung derartiger Skalen-Ontologien zu durchbrechen, beispielsweise die Auffassung, dass Diskurse um das „Globale“ den einzelnen Menschen als handlungswirksames Subjekt entmachten
(Gibson-Graham, 2006). Im praxeologischen Sinne ist entscheidend, was tatsächlich geschieht. Dabei ist alles multiskalar, denn von jedem praktischen Geschehen aus lassen sich vielfältige Bezüge herstellen, oder wie Latour es ausdrückt: „Der winzigste Aidsvirus bringt uns vom Geschlecht zum Unbewußten, von dort nach Afrika, zu Zellkulturen, zur DNS, nach San Francisco“ (Latour, 2008, 8). Aber eine „Ontologie“, wenn man es schon so nennen möchte, wird nicht dadurch „flach“, dass man die Worte „lokal“, ,regional“, „global“ oder „,mikro“, „,meso“, ,makro“ vermeidet; sie sollten nur keineswegs als geographische A priori-Kategorien angesehen werden. Es spricht nichts dagegen, ein empirisch auftauchendes Phänomen beispielsweise als ,global“ zu bezeichnen, wenn im Kontext der Untersuchung dadurch eine bestimmte Relation ausgedrückt werden kann. Die Skalenbegriffe machen durchaus Sinn, wenn sie nicht als metaphysische Setzungen der Analyse vorausgehend verstanden werden, sondern in ihrem Verweischarakter auf Erfahrungen gesehen werden, wie z. B. in politischen Prozessen oder in Planungsprozessen die Erfahrung städtischer Bedienstete und Politiker*innen, dass gewisse staatliche Gesetzgebungen bindenden Charakter haben. Die von Schatzki vorgeschlagene Differenzierung von ,large versus small“ löst damit keineswegs das Skalen-Dilemma, sondern führt stattdessen weitgehend unspezifische Kategorien ein, die zudem ein „larger" oder ,smaller" assoziieren, wodurch sich im Zweifelsfall sogar Größenvergleiche aufdrängen könnten.

Schatzki betrachtet soziale Phänomene also als Einheiten. Seine Terminologie suggeriert Abgeschlossenheit und eine gewisse Stabilität, die sich trotz beständigen Wandels einstellt und schließlich auch wieder vergeht. Dies steht im Gegensatz zur empirisch feststellbaren Gleichzeitigkeit, Widersprüchlichkeit und Multiplizität von Praxis, in der Phänomene in ihren Grenzziehungen und Identitäten immer divers und indeterminiert sind. In diesem Sinne wollen wir im Folgenden vorschlagen, sich empirisch auftauchenden Phänomenen nicht mittels eines Begriffs wie „large social phenomena“, sondern mit einer Frage zu nähern, nämlich der, ob es sich bei ihnen um eine große Sache (,big issue") handelt.

\section{Ist es eine „große“ Sache? Möglichkeiten der Analyse überindividueller Sachverhalte}

Im praxeologischem Verständnis geht es darum, ein empirisches Phänomen in seiner Stellung zwischen Struktur und Handlung zu charakterisieren und somit die Grenzen seiner Konventionalisierung und Institutionalisierung auszuloten. Hierzu soll zunächst das in der Einleitung formulierte empirische Dilemma von Konstruktivismus und Objektivismus adressiert werden. Zur begrifflichen Bestimmung empirischer Phänomene orientieren wir uns dabei an Latours Konzept des „Quasi-Objekts“. 


\subsection{Quasi-Objekte: „matters of facts“ und „matters of concern}

Latour zufolge ist Praxis durch die Entstehung von Hybriden charakterisiert. Für die Erklärung eines Phänomens, die im gesellschaftlichen Kontext auch als zufriedenstellend wahrgenommen wird, reicht weder allein die naturwissenschaftlich abgesicherte Erklärung dieses Phänomens (Realismus) noch die allein kulturwissenschaftliche Erklärung, die dieses Phänomen als ein Produkt menschlichen Handelns erklärt (Relativismus), aus. Obwohl die Wissenschaft sich vehement um die Trennung dieser Perspektiven bemüht, werden solche Hybride aus Natur und Kultur alltäglich millionenfach hergestellt. Latour bezeichnet derartige Hybriden als "Quasi-Objekte“ (Latour, 2008, 70). Quasi-Objekte sind real, weil sie zuvor konstruiert wurden. Sie eröffnen zwei Möglichkeiten eines kritischen Verständnisses bezüglich der Angemessenheit der Konstruktion. Die Behandlung von Quasiobjekten ist für Sozialwissenschaftler*innen schwierig, denn ,auch sie ,sehen doppelt‘. Für die erste Form von Kritik zählen die Objekte nicht; sie bilden nur die Leinwand, auf die die Gesellschaft ihren Film projiziert. Für die zweite sind die Objekte jedoch so mächtig, daß sie die menschliche Gesellschaft gestalten; ausgeblendet wird dabei die gesellschaftliche Konstruktion der Wissenschaften, die diese Objekte hervorgebracht haben“ (Latour, 2008, 72 f.). Der Begriff der Quasi-Objekte verweist auf die empirische Notwendigkeit, zwei Effekte zugleich zu untersuchen: einerseits die kognitive Vorgängigkeit von Konzepten und andererseits die erlebbare Faktizität der mit diesen Konzepten bezeichneten Phänomene. Praxeologische Konzepte helfen, diese doppelte Bewegung empirisch fassbar zu machen.

Die doppelte praxeologische Bewegung von realistischer und (de)konstruktivistischer Beschreibung lässt sich empirisch operationalisieren, solange kein Repräsentationsanspruch erhoben wird. Das sich in der Empirie zeigende Quasi-Objekt sollte nicht wie ein ,matter of fact", sondern als „matter of concern" behandelt werden (Latour, 2004). Demnach befragen Forschende einen Sachverhalt daraufhin, ob und inwiefern ein benennbares Objekt des sozialen $\mathrm{Zu}$ sammenhangs für die Beforschten „eine große Sache“ darstellt, in welchem Maße sie sich auf dieses Objekt einstellen, sich anpassen und zu dessen Persistenz oder Veränderung beitragen. Die praxeologische Aufhebung der SubjektObjekt-Dichotomie verlangt eine Betrachtung der gegenseitigen Ko-Produktion der Beforschten mit dem Objekt (Markt und Markteilnehmer*innen). In der Empirie stellt sich für der*die Forscher*in also die Frage, wie der zu betrachtende Zusammenhang im aktuellen situativen Setting emergiert.

Wie entstehen oder entstanden im Untersuchungsfeld bestimmte Vorstellungen von und Umgangsweisen mit dem fraglichen Phänomen? Erst die Etablierung einer gewissen kommunikativen Verbindlichkeit für bestimmte Aussagen über einen Sachverhalt und von entsprechenden darauf bezogenen Praktiken macht diesen Sachverhalt intersubjektiv erkennbar; er scheint zu existieren. In dem er benannt wird, einen Namen bekommt, tritt er als eine soziale Wirklichkeit in Erscheinung. Die wiederholt gelingende Ausführung bestimmter Praktiken der Beforschten zeugt schließlich von der Angemessenheit und Gangbarkeit bzw. Viabilität der Vorstellungen von diesem Sachverhalt, wodurch sich diese Vorstellungen schließlich etablieren. Durch diese Etablierung aber entsteht die Illusion, dass hinter den diversen Vorstellungen ein geschlossenes, wesenhaftes Objekt (,,matter of fact “) steht. Es ist insbesondere die Tatsache, dass es unter einem bestimmten Namen auftritt, die diese Reifizierung als Objekt bewirkt. In der Regel bleibt aber unerkannt, dass jede Einzelvorstellung und jede einzelne darauf bezogene Praktik das Objekt jeweils nur auf bestimmte Teilaspekte reduziert und in einer bestimmten Funktion hervorbringt. Diese Teilaspekte entsprechen der Art und Weise, wie die Beforschten von den gegebenen Bedingungen beeinflusst werden (,,concerns").

Für die empirische wissenschaftliche Analyse ist es demnach nicht angemessen, die Wesenhaftigkeit von Objekten des sozialen Lebens ergründen zu wollen. Das wäre eine Reifizierung des Objekts als ,matter of fact“. Vielmehr ist es sinnvoll für Forschende, eine Vorstellung von den Rändern, den Säumen oder den Grenzen sozial als relevant wahrgenommener (Quasi-)Objekte zu entwickeln, so wie sie durch die Betroffenheit der Beforschten (,concerns") fixiert werden. Derartige Grenzziehungen möchten wir im Folgenden als Konkretisierung der Reichweite, der Tragweite sowie der Importanz und Relevanz von Phänomenen beschreiben.

Um die Entwicklung der vorgeschlagenen Begriffe zu illustrieren, kehren wir im weiteren Verlauf wiederholt zum eingangs verwendeten Beispiel des „Marktes“ zurück. Anhand von Vignetten aus der Feldforschung zu Initiativen und Organisationen, die versuchen sich marktförmigen Praktiken zu entziehen oder diese zu verändern, zeichnen wir die Ränder, Säume und Grenzen des (Quasi-)Objektes „Markt“ nach. Die anonymisierten Beispiele sind einem in seiner Methodik praxistheoretisch ausgerichteten Forschungsprojekt entnommen, das unter der Fragestellung nach der Rolle zivilgesellschaftlicher Organisationen in Transformationsprozessen hin zu einer wachstumsunabhängigen Wirtschaft am Beispiel Stuttgart in den Jahren 2015-2019 durchgeführt wurde (Schmid, 2020). Untersucht wurden 24 Organisationen, darunter Unternehmen, Vereine und Projekte, die eine explizit soziale oder ökologische Zielsetzung, bis hin zur Veränderung der wirtschaftlichen Rahmenbedingungen selbst, verfolgen. Ergänzt durch Interviews, war die Forschung primär ethnographisch ausgerichtet, sodass die im Folgenden angeführten Vignetten als Zusammenfassung der im Kontext der Feldforschung gemachten und dokumentierten Beobachtungen zu lesen sind. Während die darin geschilderten Sachverhalte direkt übernommen wurden, wurden die Ausformulierungen der Beobachtungen dem vorliegenden Artikel angepasst. Aufgrund des rein illustrativen Charakters der Beispiele wird an dieser Stelle auf einen separaten Methodenteil ver- 
zichtet. Details zum Forschungsprojekt und seiner Methodologie können nachgelesen werden bei Schmid (2020).

\subsection{Reichweite}

Mit Reichweite ist ein Einflussgebiet im Sinne der AusWirkung und Aus-Dehnung eines von den Beforschten wahrgenommenen Sachverhaltes gemeint. Dies kann einerseits von dem*der Forscher*in ${ }^{3}$ zunächst konkret räumlich ausgedehnt gedacht werden und bezeichnet entsprechend das Gebiet, in welches der Einfluss eines Phänomens in die Praktiken der Beforschten hineinreicht. Beforschte Personen versuchen demnach die Reichweite der ihre Aktivitäten betreffenden Phänomene (z. B. den Markt) möglichst genau zu bestimmen, um sich dann wahlweise innerhalb oder außerhalb der angenommenen Konventionen dieses Phänomens (z. B. Preiskonkurrenz) zu verorten. Innerhalb, wenn es hilfreich erscheint (weil andere Anbieter günstiger anbieten), auBerhalb aber, wenn beispielsweise Konkurrenz zu mächtig wird, Regularien bestimmte Aktivitäten einschränken oder bestimmte Praktiken innerhalb der Reichweite des Phänomens als nicht sinnvoll gelten. Mit Konventionen sind hierbei Annahmen, Lehrmeinungen, in gewisser Hinsicht auch „common sense“ gemeint, also das, was „man“ denkt bzw. was nicht hinterfragt wird. Bei der Reichweitenbestimmung antizipieren die Beteiligten (Marktteilnehmer*innen) demnach die Grenzen des Phänomens und wenden versuchsweise Praktiken an, um diese Konstruktion auf ihren realistischen Gehalt zu prüfen. Gelingt diese Reichweitenfixation auf kommunikativer Ebene, dann emergiert das Phänomen für die Beforschten. In der Folge werden die projektierten Vorhaben nun entweder als umsetzbar oder unmöglich durch die Beteiligten erkannt.

Entsprechend werden die Beteiligten ihre Praktiken so gestalten, dass sie sich partiell außerhalb bestimmter Konventionen befinden, ohne dabei aber die Konventionen in Gänze abzulehnen. Diese Konkretisierung der Reichweite des Phänomens eröffnet den Beforschten eine für ihre Aktivität charakteristische Nische. Diese Nische kann als lustvoller Antrieb erfahren werden und wird oft rückwirkend als Gründungsmythos verklärt, wie z. B. im „Mythos Garage“ (feuilletonistisch dazu vgl. Schmieder, 2014). Angetrieben vom Willen, ein Problem zu lösen, kann sich eine Kreativität des Handelns entfalten (Joas, 1992). Die Übertretung einer ursprünglich für gegeben angenommenen Reichweite kann als Pionier-Erfahrung gelten. Es ist das kreative Element von Praktiken - der Bewegung ,outside the box" -, welches im Kontext der „vita activa“ (Arendt, 1981) als an-

\footnotetext{
${ }^{3}$ Die hier etwas künstlich anmutende Differenzierung zwischen Beforschten und dem*der Forscher*in dient dem Verständnis der drei aufgeworfenen Konzepte. Natürlich ist den Autor*innen bewusst, dass auch wissenschaftliche Praktiken eine Koproduktion von Forschenden und Beforschten erzeugen und dass dieser Umstand ebenfalls einer Reflexion bedarf.
}

genehmes Erlebnis oder als eine gelingende Erfahrung (Dewey, 1980[1934]) beschrieben werden kann.

Nicht nur die Quasi-Objekte müssen in der Reichweite bestimmt werden. Zur erfolgreichen Ausgestaltung einer Praktik (ökonomische Aktivität) muss deren Reichweite bestimmt werden, d. h., die Begrenzung oder etwa Eindämmung durch das Quasi-Objekt (Markt) muss schrittweise erfasst und vermessen werden. Dies findet sich in alltagsweltlichen Redewendungen der Beforschten wie „Fangen wir erst mal klein an“ oder „Einigen wir uns erstmal auf ein paar Grundsätze“. Mit Grund könnte der*die Forscher*in hier einen „Boden“ und eine pragmatische Territorialität assoziieren, von welcher etwas ,aus-geht“". Bei gemeinschaftlichen Aktivitäten möchten die Beforschten etwas aus-gehen lassen, einen Einfluss begründen und möglichst weitreichende Folgen zeitigen. Dies kann aber nur gelingen, wenn einschränkende Einflüsse minimiert werden, Beforschte sich also möglichst außerhalb der Reichweite ähnlicher schon etablierter Praktiken - z. B. in ökonomischen Nischen - positionieren.

Es geht um die Erfahrung des Gestaltens und des Entdeckens als ein Hinausreichen in die Welt. Als ein Erleiden von Rückschlägen, ein Experimentieren und schließlich um eine Freude am praktischen Erfolg. Die abschließende Freude daran, dass etwas „läuft“" bzw. gelingt, und die Beforschten schließlich verstanden zu haben glauben, warum das so ist, konsolidiert eine Erfahrung (vgl. das , flow"-Erlebnis, Cszíkszentmihályi, 2004; Dewey, 1980[1934]). Es vergrößert die Wahrnehmung der Reichweite der angewendeten Praktiken und kann affektiv als befriedigend und befreiend empfunden werden. Dieser Wahrheitseffekt der Bestätigung der Selbstwirksamkeit kann sich insbesondere in „communities of practice " in räumlicher, moralischer, ästhetischer Hinsicht als besondere oder kreative Atmosphäre (z. B. im Sinne einer ,industrial atmosphere“ nach Alfred Marshall, vgl. Ravix, 2012) niederschlagen.

Das Experimentieren mit unterschiedlichen Praktiken dient den Beteiligten zur Objektivierung der Grenzen eines Phänomens, welche im Falle der Reichweite räumliche, ästhetisch-metaphorische und moralisch-rechtliche Grenzen sein können. Die Reichweite eines Phänomens ist stets Anlass für vielfältige Spekulationen, derer man eben nur metaphorisch Herr wird. Dies zeigt sich beispielsweise darin, dass kapitalistische Marktdynamiken gerne mit der Metapher der „unsichtbaren Hand“ beschrieben werden. Die Metapher dient dabei als quasi-magisches Element der Objektivierung. Der Wirtschaftswissenschaftler Beckert (2016) hat auf die Kraft von Vorstellungsbildern und Erzählungen im Marktgeschehen hingewiesen. In rechtlicher Hinsicht kann die Reichweite entweder prohibitiv, also durch Verbote, Tabus, Paternalismus oder permissiv durch Legalisierung eingehegt (sprichwörtlich: durch den ,langen Arm des Gesetzes") oder erweitert werden.

Gesellschaftlich wird der potentiellen Uferlosigkeit der Reichweite eines Phänomens mit institutionalisierten Grenzziehungen als Einfassung und Umrandung begegnet. Die 
Furcht vor einer ungehinderten, unbemerkten Ausbreitung eines Phänomens stellte schon immer ein Schreckensszenario dar, prototypisch verkörpert im berühmten Schmetterlingseffekt (Lorenz, 1995), der im Prinzip nichts Anderes zum Ausdruck bringt, als die Unmöglichkeit zu einer rationalen Abschätzung der Reichweite von Phänomenen oder des eigenen Tuns zu kommen. So erklärt sich die Überraschung von Beforschten („Ich hätte nicht geahnt, dass es einmal so weit kommt"), wenn sie mit unerwarteten Konsequenzen ihres Tuns konfrontiert werden. Die biologische Metapher der „Viralität“ (Sampson, 2012) verkehrt im Zeitalter der globalen Digitalisierung genau diese Angst in eine Chance jenseits bestehender Konventionen eines Phänomens, sich anfangs quasi unsichtbar ausbreiten zu können, um dann unvermittelt mit hoher Popularität auf den Plan zu treten.

Andererseits haben Beteiligte oft ein erstaunlich deutliches Verständnis über die mögliche Reichweite eines Phänomens, die keineswegs auf vorher beschriebenen Auslotungsversuchen einer konkreten Reichweite (z. B. „Marktdurchdringung“, „Benchmark-Analyse“) beruhen. Bei den Beforschten kann dann eine Als-ob-Haltung (Beckert, 2016, $10)$ festgestellt werden. Beckert $(2016,9)$ hat dies als ,fiktive Erwartungen“ bezeichnet. Dies ist für Marktkapitalismus insofern von Bedeutung, als dass es vorkommt, dass Risiken eingegangen werden, die eigentlich nicht kalkulierbar sind. Es ist keine bloße Risikofreudigkeit im Sinne eines ,Wir schauen mal, was passiert“, sondern mittels Kalkulation, Abwägung und Berechnung werden Unsicherheiten (vermeintlich) eingedämmt. Die prognostizierte Reichweite stellt sich hier als „Blase“ (,,bubbles") dar, die dann in kritischer Zuspitzung, d. h. in einer Krise, platzen kann (zur Soziologie von bubbles vgl. Tapia, 2004; Goodnight und Green, 2010; Abolafia, 2010).

Anschaulich wird das bisher Gesagte in einem konkreten empirischen Beispiel aus der Forschung zu alternativwirtschaftlichen Praktiken:

Paul, ein Sozialunternehmer und Tüftler, entwickelt, konstruiert und vertreibt langlebige, reparierbare und möglichst fair gehandelte Leuchtmittel, die dem Wegwerfmodell und der Kostenexternalisierung herkömmlicher Produkte entgegenstehen. Ein weitgehend funktionstüchtiger Prototyp existiert bereits. Über ein Netzwerk steht er in Kontakt mit einer Gruppe um Andreas, die sich mit der Entwicklung autarker Wohnhäuser auseinandersetzt. Paul erkennt in diesem Projekt seine eigenen Interessen wieder. Inspiriert bietet er seine Unterstützung an und übernimmt die Installation von Leuchtmitteln beim Prototyp. Im Laufe dieser Kooperation gibt es weder Verträge noch findet ein Austausch von Geld statt. Vielmehr zeichnen sich diese Austauschbeziehungen durch freiwilliges Geben und Vertrauen aus. Obwohl beide Projekte durch Organisationen rechtlich als Un- ternehmen formiert sind, bewegen sich die Praktiken der Zusammenarbeit zunächst außerhalb der Reichweite des ,klassischen“ Marktes.

An diesem Beispiel sozialökologisch ausgerichteter Unternehmungen lassen sich Austauschbeziehungen beobachten, die nicht auf Äquivalententausch (Praktiken des Tauschens), sondern auf freiwilligem Geben, d. h. am intrinsisch motivierten Mitwirken am Projekt des Anderen und vor allem auf Vertrauen, basieren (Praktiken des Schenkens, Helfens, der Kooperation etc.). Im hier vorgebrachten Beispiel positionieren sich die Beforschten bewusst außerhalb der Reichweite des „formalen Marktes“. Formell kann das obige Beispiel als ein Übergang vom Äquivalententausch zur freiwilligen Gabe analysiert werden. Durch die Verschiebung von Praktiken des Tauschens zu Praktiken des Gebens entziehen sich die Beforschten der rechtlichen, moralischen, rationalen und materiellen Reichweite des Phänomens „Markt“. Die Protagonisten des Beispiels treten ohne die Bedingungen und Unterstützungen rechtlicher Rahmenbedingungen miteinander in Beziehung und bewegen sich damit außerhalb der Reichweite bürokratischer Praktiken (die in Schatzkis Terminologie als Teil des ,large social phenomena"-Staats beschrieben werden könnten). Moralisch treten sich die Beforschten nicht als Dienstleister und Dienstnehmer gegenüber, wobei letzterer mittels Geld seine „Schuld“ vergelten könnte, sondern als Teile einer (nicht definierten) Gemeinschaft, in der Hilfe (statt Arbeit) nach Bedarf und Möglichkeiten gegeben (statt geleistet) werden (ähnlich beziehen sich auch Mitglieder von Familienkreisen weitgehend außerhalb der Reichweite von Praktiken des Äquivalententausches aufeinander). Die Beforschten bewegen sich zudem außerhalb der Marktrationalität des Geldverdienens, indem Teilhabe und Unterstützung gemeinsamer Ziele oder die einfache Tatsache des Miteinander-etwas-Machens im Mittelpunkt stehen. Zuletzt entziehen sich die Protagonisten der Reichweite des Marktes als Mechanismus der Ressourcenallokation. Da die Zusammenarbeit finanziell nur schwer hätte vergolten werden können, ermöglicht erst das Agieren außerhalb der praktisch angenommenen Reichweite des Phänomens Markt die Zusammenarbeit als solche.

Die beobachteten Praktiken finden in einem Grenzbereich des ,klassischen“ Marktes statt und bringen diesen dadurch in ständige Bewegung. Das Quasi-Objekt des ,klassischen Markts" dringt

in die Praktiken alternativwirtschaftlicher Organisationen ein. Dies geschieht erratisch, d. h. nicht einem festgelegten Muster folgend. Dies tritt beispielsweise ein, wenn Organisationen kurzfristig Liquidität brauchen und sich aus Austauschbeziehungen (temporär) zurückziehen, die nicht oder nur bedingt monetär vermittelt sind. Sie fokussieren dann eher auf solche, die stärker gemäß ,klassisch marktförmigen" Tauschlogiken organisiert sind. Somit findet eine Bewegung von Praktiken des Gebens und des freien Kooperierens hin zu Praktiken des geldvermittelten Tauschens statt. 
Gleichzeitig reichen zentrale Praktiken einiger alternativwirtschaftlicher Organisationen, wie beispielsweise Reparieren, Selbstmachen oder Teilen (von Wissen, Artefakten etc.), in maßgeblich durch Praktiken „marktförmigen“ Produzierens, Konsumierens und Tauschens vermittelte Räume hinein. Beispielsweise spiegelt sich die zunehmende Verbreitung von Praktiken des Reparierens - insbesondere im Rahmen sog. Repaircafés und Offenen Werkstätten - in der Verschiebung, Modifikation und Adaption globaler Wertschöpfungsketten wie beispielsweise im Falle des (modular konstruierten) Fairphones wider. Auch Praktiken des Spendens (expendere: auszahlen, ausgeben) ermöglichen das Ausbrechen aus Konventionen dessen, was als ,klassische Marktförmigkeit" angenommen wird. Diese bringen in zweiter Instanz aber neue Quasi-Objekte hervor, wie beispielsweise die Spendenmärkte der humanitären Hilfe (Silk, 2004).

Mit der Analysekategorie Reichweite können Forschende also danach fragen, wie die Beforschten die materiellen, diskursiven und moralischen Grenzen eines Phänomens bestimmen und gestalten. Anstatt also Markt als gegebenes und damit verdinglichtes Phänomen anzunehmen, richtet sich der hier vorgeschlagene Blick darauf, wie weit die mit dem Phänomen Markt assoziierten Praktiken des geldvermittelten Äquivalententausches in die konkreten empirischen Zusammenhänge hineinreichen. Ebenso kann von Interesse sein, alternativen Formen des wirtschaftlichen Miteinanderin-Beziehung-Tretens $\mathrm{zu}$ folgen und $\mathrm{zu}$ untersuchen, wie weit die Praktiken des Teilens, Schenkens, Helfens in weitere soziale Zusammenhänge hinausreichen. Damit werden die Grenzen und Säume von Quasi-Objekten als „,matter for concern “ sichtbar, ohne diese als ,matter of fact “ festzuschreiben.

\subsection{Tragweite}

Unter Tragweite wird eine Perspektive auf die folgenreiche Auswirkung von Praktiken verstanden. Dabei stellt sich für den*die Forscher*in die Frage, was das Phänomen den Beforschten abverlangt und wie weit es sie trägt, wenn sie sich darauf einlassen. Ebenfalls aber auch bezüglich der Reziprozität, d. h., es stellt sich die Frage, was es für Andere bedeutet, wenn sich jemand auf ein Phänomen einlässt (z. B. die Übernahme einer verantwortungsvollen Aufgabe, eine neue berufliche Position). Solche Phänomene tangieren dabei die Sphäre der Moral und Ethik (Leitfrage: Welche Verantwortung trägt man für andere Personen, Tiere und die Umwelt?) und der Politik (Leitfrage: Welche Verantwortung ist man bereit zu übernehmen und zu tragen?).

Die Tragweite eines Phänomens lässt sich ferner als ihre transformative Wirkmächtigkeit beschreiben. Erfordert das Phänomen eine Veränderung oder Verwandlung der Beteiligten? Dies wird insbesondere deutlich, wenn in bestimmte Aufgaben „hineingewachsen“ werden muss oder erwartet wird, dass eine Persönlichkeitsentwicklung für die Übernahme bestimmter Aufgaben erwartet wird. Hierbei geht es beispielsweise um Führungsaufgaben oder die Übernahme einer vereidigten Tätigkeit (Verfassungsschwur, hippokratischer Eid etc.). Die Tragweite von Phänomenen des Lebens und des Tods zeigen sich in Praktiken der Geburts- oder Sterbehilfe oder in Praktiken der Unfallvermeidung. Mittels dieser extremen oder alltagsweltlich hervorgehobenen Praktiken wird die transformative Wirkmächtigkeit der Tragweite besonders deutlich. Ähnlich gilt dies z. B. für Praktiken der Investition von Wagniskapital, was ggf. - metaphorisch schon als vermeintlich metaphysische Tragweite angedeutet - durch ,business angels“ abgemildert werden kann.

Bei der Tragweite geht es ferner um praktische Sinnstiftung. Praktisch ist diese Sinnstiftung, da sie auf ein Ziel hin ausrichtet und als teleo-affektive Orientierung dient. Dies kann ein Gedanke sein, der „trägt“, oder eine Absicht, die sich auf eine „Leuchtturm-Initiative“ ausrichtet, die mit Praktiken in Verbindung steht. Dazu eine allegorische Erläuterung: Um im Winter an das andere Ufer eines zugefrorenen Sees zu kommen, ohne diesen zu umrunden, muss die Dichte der Eisdecke geprüft werden. Zur Feststellung der Tragweite (im Bild: eines zugefrorenen Sees) muss die Tragfähigkeit (im Bild: die Eisdecke) eines Ziels (im Bild: an das andere Ufer kommen, ohne den See zu umrunden) bestimmt werden. Nun lässt sich beobachten, wie die Beforschten die Tragweite eines Phänomens abschätzen. Scheint es ein gefährliches Spiel zu sein, wagen sie sich nicht zu weit raus oder zeigen sich mutig, „fassen sich ein Herz" und bekennen sich zur Offenheit des Ausgangs. Zweifel, Unschlüssigkeit und Unglauben gefährden die Tragweite eines Vorhabens. In der Forschungspraxis stellt sich folglich die Frage, ob Modelle oder Interpretationen für die Beteiligten tragen. Entscheidend ist dabei, was von den Beforschten als ,fundamental“, „solide“ oder ,profund“ angesehen wird. Konzepte, Pläne und Interpretationen werden auf ihre Tragfähigkeit hin ,abgeklopft“. Dies gilt ebenso, wenn beispielsweise die Tragweite des „Marktes“ angezweifelt wird. „Markttauglichkeit" beschreibt die Fixation der Tragweite eines Produkts oder Prototypen.

Die Tragweite ist ein wichtiges Motiv in narrativen Praktiken, die alternativwirtschaftliche Organisationen begleiten und motivieren, wie beispielsweise im Ausspruch ,think global, act local" zu vernehmen ist. Der (an konkreten Orten stattfindenden) Ausführung von Praktiken wird eine große Tragweite im Sinne einer Auswirkung zugeschrieben, die diese auf als global angenommene Phänomene (z. B. Klimawandel, Biodiversität, Hungersnöte etc.) hat. Tragweite kann auch die teils widersprüchliche Spannung zwischen Dezentralisierung, Regionalisierung, Lokalisierung auf der einen Seite und der Adressierung globaler Problemstellungen auf der anderen Seite beleuchten. Praktiken der Selbstversorgung erlauben es beispielsweise, sich von öffentlichen Infrastrukturen unabhängig(er) zu machen. Meist geht es dabei um Minimierung der mit der Versorgung verbundenen Kosten, d. h. einer Abgrenzung von geldbasierten Austauschpraktiken, für 
die letztendlich an Praktiken des Arbeitsmarktes teilgenommen werden muss.

Betrachten wir dies anhand einer Szene aus der Empirie:

Zusammen mit Paul verfolgt die oben schon erwähnte Gruppe um Andreas das Ziel, durch ein autarkes Haus einerseits Kreisläufe zu schließen, die es erlauben, alltägliche Grundbedürfnisse ressourcenschonend(er) zu decken. Zum anderen geht es darum, sich von öffentlichen Infrastrukturen und damit der durch sie vermittelten Kontrolle über die Bereitstellung von „Lebensmitteln“ (Wasser, Strom, Wärme, Nahrung. ..) unabhängig(er) zu machen. Andreas selbst wohnt seit einigen Monaten im Prototyp und nimmt damit in seiner alltäglichen Praxis - Kochen, Essen, Duschen, Reparieren, Entspannen, Lesen, Kommunizieren nur sehr bedingt an marktförmigen Praktiken teil. Die Selbstversorgung erlaubt es Andreas, sich aus vielen marktvermittelten Austauschpraktiken und den damit verbundenen globalen Wertschöpfungsketten zurückzuziehen. Alternativ- und subsistenzwirtschaftliche Praktiken tragen ihn in Bezug auf einige seiner alltäglichen Bedürfnisse. Jedoch reichen gesetzliche Vorschriften bis in die alternativwirtschaftliche Selbstversorgung hinein. Andreas hat viel mit den Behörden zu kämpfen, die Vorschriften durchsetzen wollen, die besagen, dass Wohnhäuser an die öffentliche Wasserver- und entsorgung angeschlossen sein müssen.

Alex, ein*e Student*in im Bereich Wassermanagement und Sympathisantin des Projektes, setzt sich derzeit im Rahmen der Masterarbeit mit genau diesen Vorschriften auseinander. Gemeinsam mit der Gruppe um Andreas erhofft Alex, sich durch Messungen der Wasserqualität der hauseigenen ökologischen Kläranlage, die zusammen mit dem Gesundheitsamt durchgeführt werden, einen Präzedenzfall zu schaffen. Ein solcher könnte eine beachtliche Tragweite entfalten und exemplarisch für die Möglichkeit anderer Versorgungspraktiken einstehen.

Scheinbar auf das Lokale begrenzte Praktiken der Beforschten kommen vor allem durch ihre Abwesenheit in größeren Zusammenhängen zum Tragen. Praktikenzusammenhänge, die die globalen Märkte reifizieren, sind von der (massenhaften) Ausführung entsprechender Praktiken abhängig. Staatliche Bürokratien festigen diese Praktikenzusammenhänge, wodurch diese zur Norm werden und zu quasinatürlicher Voraussetzung von Märkten geworden sind. Die Beschränkungen sind so lange unsichtbar, bis alternative Praktiken deren Tragweite in Frage stellen. Mögliche Präzedenzfälle der gelungenen Aussetzung dieser Beschränkungen (z. B. durch Widerlegung gängiger Vorstellungen von der hygienesichernden Notwendigkeit öffentlicher Versorgungssicherheit) können dann ihrerseits wiederum beachtliche Tragweite entfalten.

Mit der Analysekategorie Tragweite fragen die Forschenden nach den Auswirkungen von Phänomenen und wo die mit ihnen verbundenen Praktiken hinführen. Der Blick wird darauf gerichtet, wie weit und wohin die Praktiken die Beforschten tragen. In unserem Fallbeispiel entfalten alternative Versorgungspraktiken eine hohe Tragweite, da sie die Beforschten nicht nur in der Befriedigung ihrer alltäglichen Bedürfnisse tragen, sondern für diese auch sinnstiftend sind. Die Abwendung von markt- und staatsförmigen Versorgungsstrukturen entstammt der Überzeugung, dass diese nicht dauerhaft tragfähig sind.

Gleichzeitig wird hier eine enge Verbindung mit der Analysekategorie der Reichweite deutlich. In der Dynamik der dargestellten empirischen Zusammenhänge wird die Tragweite alternativer Versorgungspraktiken durch das HineinReichen von Praktiken der bürokratischen Kontrolle in Frage gestellt. Zwar gelingt es den Beforschten, Teile ihrer alltäglichen Versorgung mit Energie, Wasser und Lebensmitteln in einer sich technisch und wirtschaftlich tragenden Zirkularität zu organisieren. Jedoch wird diese wiederum durch die Reichweite bürokratischer Kontrollpraktiken destabilisiert. Auch ohne die polizeiliche Durchsetzung der Vorschriften wird die alternative Versorgungspraxis von Andreas und Co. dadurch unterminiert, dass sie jederzeit wieder wegfallen könnte.

\subsection{Importanz und Relevanz}

Es lässt sich ferner beobachten, dass sich bestimmte Phänomene Forschenden ,als relevant“ aufdrängen. Zum Beispiel dann, wenn sich eine Finanzlücke auftut, die eine sofortige Reaktion erfordert. Es treten Notwendigkeiten auf, die sich quasi wie ,,von alleine“ als Selbstverständlichkeiten präsentieren. In einem empirischen Beispiel zeigt sich dies wie folgt:

Sarah, Asaf und Kim sind Ingenieure, die Andreas und sein Team vor allem in Bezug auf die Regulationsautomatisierung von Wasser-, Stromund Nährstoffkreisläufen unterstützen. Wie Andreas sind auch sie begeistert vom Projekt und sehen darin weit mehr als einen formellen Arbeitsauftrag an ihr Ingenieurbüro. Für Teile ihrer Arbeit stellen sie Rechnungen, andere werden als Freundschaftsdienst angesehen und in ihrer Freizeit durchgeführt. Andreas und sein Team sind froh, vielseitige Unterstützung zu bekommen - erst von Paul, dann auch von Sarah und ihren Mitstreitern -, da die Finanzmittel chronisch knapp sind. Gleichzeitig müssen Sarah, Asaf und Kim den Betrieb ihres Ingenieurbüros sicherstellen, sodass Freundschaftsdienste nur in einem begrenzen Rahmen ge- 
leistet werden können. Im Oktober stellten sie fest, dass durch Vorleistungen, ausstehende Rechnungen und noch nicht abgeschlossene Projekte ein zu geringer Puffer vorhanden war. Die Faktizität dieser Finanzlücke erforderte eine sofortige Priorisierung von besser bezahlten Aufträgen, sodass die Unterstützung zunächst eingestellt wurde.

Die Relevanz eines Phänomens erfordert in dringlicher Weise Aufmerksamkeit und eine Neuausrichtung. Eng damit verbunden, die Importanz. Darunter verstehen wir bereits priorisierte Relevanz. Importanz hat insofern mit Macht zu tun, als dass Priorisierungen zumeist vermittels hierarchisierender Praktiken vorgenommen werden. Es ist machtvoll, weil es soziale Organisation erfordert und es oft unter Zeitmangel zur machtvollen Durchsetzung von Relevanz kommt. Deswegen sind Kinder bspw. ungeeignete „Marktteilnehmer", weil sie noch nicht gut zwischen Relevanzen unterscheiden können. Der praktische Umgang mit Relevanz ist erlernbar. Dies ist für Forschungsprozesse von immenser Bedeutung: Die Relevanz drängt sich zwar auf und gibt sich zu erkennen, aber muss praktisch in Importanz verwandelt werden. Die Importanz verweist darauf, dass man es als durchaus abzählbare Größe handhaben kann. Davon künden die To-do-Listen und Software-Anwendungen zum Projektmanagement.

Im Vorgehen alternativwirtschaftlicher Organisationen spielt das Bestimmen von Relevanz oft eine (überlebens-)wichtige Rolle. Häufig werden Organisationen mit dem Widerspruch konfrontiert, dass sich mit der Teilnahme an Praktiken, die für sie nicht erstrebenswert erscheinen (z. B. aufgrund ihrer sozialen oder ökologischen Auswirkungen), Geld verdienen lässt, welches wiederum zum Bestehen der Organisation (und Finanzierung des eigenen Lebensunterhaltes) benötigt wird. Gleichzeitig bedeuten Praktiken des Helfens und Unterstützens (z. B. wenig zahlungskräftiger Kunden, Organisationen), der Kooperation, der Internalisierung (von sonst externalisierten) Kosten in der Produktion, des Einkaufens von als organisch/fair gehandelt zertifizierten Produkten etc. oft erhöhte Kosten, die nur bedingt kompensiert werden können. In diesem Spannungsfeld lassen sich Hybridisierungen, Kompromisse, Abwägungen etc. beobachten. Dies zeigt sich beispielsweise in Organisationen, die mit zahlungskräftigen Konzernen (deren Praktiken ansonsten als ausbeuterisch kritisiert werden) kooperieren und mit den dadurch erwirtschafteten Finanzmitteln wiederum den Betrieb für Individuen bzw. Organisationen, die weniger zahlungskräftig sind und eine sozialökologische Ausrichtung haben, quersubventionieren.

Mit den Analysekategorien Relevanz und Importanz fragen Forschende nach Bedeutung und Dringlichkeit von Phänomenen. Der Blick wird darauf gerichtet, in welcher Wichtig- und Dringlichkeit bestimmte Sachverhalte in den untersuchten empirischen Zusammenhängen auftreten. In unserem Fallbeispiel drängt sich die ansonsten weitgehend in den Hintergrund geratende bezahlte Arbeit plötzlich als dringlich in den Vordergrund.

Diese Priorisierung lässt wiederum Momente der Tragweite und Reichweite erkennen. Marktförmige Austauschbeziehungen reichen erratisch in die Praktiken der Organisation hinein und drängen sich auf, wodurch Grenzen der Tragweite sichtbar werden. Während marktförmige Praktiken die Protagonisten nicht tragen, da sie durch Konkurrenz und Kostenexternalisierung nur bedingt die Umsetzung kooperativer und nicht-extraktiver Praktiken zulassen, ist es ihnen nur bedingt möglich, sich diesen zu entziehen. Mit der Relevanz bezahlter Arbeit konfrontiert, erscheint wiederum das Projekt von Andreas und Co. als Möglichkeit, sich dem Phänomen ,Markt“" zu entziehen und durch die Tragweite alternativer Versorgungspraktiken andere Formen des Wirtschaftens zu praktizieren.

\section{Konklusion und Ausblick}

Unsere Beispiele aus Transitionsinitiativen haben gezeigt, wie sich ,Markt“" vermittelt durch Praktiken auf unterschiedliche Arten und Weisen intersubjektiv konkretisieren kann. Dadurch, dass wir die Betroffenheiten als real ansehen, gelingt uns eine Erklärung, warum das Objekt, von dem Betroffenheit ausgelöst wird, auf eine bestimmte Art und Weise konstruiert wird. Transitionsinitiativen müssen neue Grenzziehungen vornehmen, die allen Beteiligten (z. B. Kunden, Behörden) intelligibel und gleichzeitig erlebbar sind. Es entsteht eine neue Erfahrung, die einen Namen bekommt (Dewey, 1980[1934]). Zur Konsolidierung dieses neuen Sachverhaltes ist es notwendig, dass alle Beteiligten diesen nicht nur verstehen, sondern ihn auch aus ihrer Perspektive heraus für existent halten, sich praktisch auf ihn einlassen und ihn emotional für wichtig empfinden. Ein neuer „Markt“ (z. B. für reparaturfähige Leuchtmittel) muss nicht nur verständlich sein, sondern auch praktisch als weitreichend, tragfähig, klug oder verderblich, auf jeden Fall aber als einflussreich, relevant und deshalb wichtig erlebbar funktionieren.

Damit wären wir beim Ausgangspunkt unserer Betrachtung. Soziale Phänomene lassen sich nicht praktikenontologisch beschreiben. Sie emergieren von klein bis groß, von ,large“ bis „small“, nicht als Tatsachen, sondern als Betroffenheit(en) (,concerns “). Deswegen können sie nicht nominalistisch auf Struktureigenschaften verkürzt werden. Sie können praxeologisch und phänomenologisch in ihrem konkreten Erscheinen oder Auftreten erfasst werden, aber nicht prinzipiell auf ontologische Prinzipien zurückgeführt werden. Phänomene sind niemals von gleichartiger Beschaffenheit, sondern jeweils singulär. Sie emergieren vielleicht auf gleichartige Weise. Schlüssig ist demnach aber nicht die Ontologie, die ein für alle Mal das Wesen von Phänomenen festlegt, sondern die Betrachtung verschiedener prozesshafter Aspekte, die allen Hervorbringungen von Phänomenen zu Grunde liegen, wie dies die Praktiken der Abschätzung 
von Reichweite, Tragweite, Importanz und Relevanz sind. Sie werden in der sozialen Praxis alltäglich millionenfach durchgeführt und sind somit Ausdruck einer sozialen Intelligenz. Entsprechend stehen wir dem von Schatzki vorgeschlagenen Begriff der ,large social phenomena“ sehr kritisch gegenüber. Hingegen sind die Tätigkeiten der Konkretisierung von Reichweite, Tragweite, Importanz und Relevanz eines wahrgenommenen oder behaupteten Phänomens empirisch feststellbare Verfahrensweisen, die praxeologisch schlüssig menschliches Tun weder als intentional individualistisch noch als strukturdeterminiert darstellen. Vielmehr wird der begrenzte Spielraum des Subjekts deutlich, in dem es sich experimentierend in den alltäglichen Begrenzungen Ellbogenfreiheit verschafft.

Mit diesem offen gehaltenen, explorativen Vokabular lösen wir das in der Einleitung aufgeworfene Dilemma zwar nicht auf, aber wir ermöglichen Forschenden, sich reflektiert mit den von ihnen untersuchten Phänomenen hinsichtlich deren Reichweite, Tragweite, Importanz und Relevanz auseinanderzusetzen. So kann der Kreislauf von Konstruktion, entsprechenden Handlungen und erneuter Reproduktion der Verhältnisse hinterfragt und aufgelöst werden. Mit unserem Vorschlag sind zwei Einladungen verbunden. Zum einen erhoffen wir uns, dass die Dienlichkeit der angebotenen Analysekategorien zur Erfassung sozialer Phänomene in weiterer praxistheoretischer Feldforschung überprüft, verfeinert und ggf. ergänzt wird. Dies sollte im besten Fall über wirtschaftssoziologische und -geographische Fragestellungen hinausgehen. Für den konkreten Forschungsgegenstand erscheint uns die konzeptionelle Weiterentwicklung dieser Begriffe möglich. So deuten unsere explorativen Erkundungen an, dass es möglich wäre, eine Praxistheorie ökonomischer Nischen $\mathrm{zu}$ entwickeln, die jenseits apriorischer Letztelemente wie „Markt“ einzig auf Tun der „vita activa“ fokussieren kann.

Datenverfügbarkeit. Für diesen Artikel wurden keine Datensätze genutzt.

Autorenmitwirkung. Alle Autor*innen waren zu vergleichbaren Teilen an der Konzeptionalisierung, Umsetzung und Überarbeitung des Artikels beteiligt.

Interessenkonflikt. Die Autor*innen erklären, dass kein Interessenkonflikt besteht.

Danksagung. Wir bedanken uns bei Benedikt Korf und drei anonymen Begutachter*innen für ihre hilfreichen Kommentare zu früheren Versionen des Artikels.

Finanzierung. This research has been supported by the German Research Foundation (DFG).
Begutachtung. This paper was edited by Benedikt Korf and reviewed by three anonymous referees.

\section{Literatur}

Abolafia, M. Y.: The institutional embeddedness of market failure: Why speculative bubbles still occur, in: Markets on Trial: The Economic Sociology of the U.S. Financial Crisis, edited by: Lounsbury, M. und Hirsch, P. M., Research in the Sociology of Organizations, Vol. 30, Part B, Emerald Group Publishing, Bingley, 177-200, 2010.

Arendt, H.: Vita activa, oder: Vom tätigen Leben, Piper, München, 1981.

Aspers, P.: Theory, Reality, and Performativity in Markets, Am. J. Economics Sociol., 66, 379-398, https://doi.org/10.1111/j.15367150.2007.00515.x, 2007.

Beckert, J.: Imagined Futures: Fictional Expectations and Capitalist Dynamics, Harvard University Press Cambridge, London, 2016.

Berndt, C. und Boeckler, M.: Geographies of circulation and exchange. Constructions of markets, Prog. Hum. Geog., 33, 535551, https://doi.org/10.1177/0309132509104805, 2009.

Cohen, D.: Between perfection and damnation: The emerging geography of markets, Prog. Hum. Geog., 42, 898-915, https://doi.org/10.1177/0309132517729769, 2017.

Csíkszentmihályi, M.: Flow, Klett-Cotta, Stuttgart, 2004.

Dewey, J.: Kunst als Erfahrung, Suhrkamp, Frankfurt a.M., 1980(1934).

Dörfler, T., Graefe, O., and Müller-Mahn, D.: Habitus und Feld: Anregungen für eine Neuorientierung der geographischen Entwicklungsforschung auf der Grundlage von Bourdieus ,Theorie der Praxis“, Geogr. Helv., 58, 11-23, https://doi.org/10.5194/gh58-11-2003, 2003.

Everts, J., Lahr, M., and Watson, M.: Theories of practice and geography, Erdkunde, 65, 323-334, https://doi.org/10.3112/erdkunde.2011.04.01, 2011.

Geiselhart, K., Winkler, J., und Dünckmann, F.: Vom Wissen über das Tun - praxeologische Ansätze für die Geographie von der Analyse bis zur Kritik, in: Praktiken und Raum. Humangeographie nach dem Practice Turn, Schäfer, S. und Everts, J., transcript, Bielefeld, 21-76, 2019.

Gibson-Graham, J. K.: The end of capitalism (as we knew it). A feminist critique of political economy, University of Minnesota Press, Minneapolis, 1996.

Gibson-Graham, J. K.: Postcapitalist Politics, University of Minnesota Press, Minneapolis, 2006.

Goodnight, T. G. und Green, S.: Rhetoric, Risk, and Markets: The Dot-Com Bubble, Q. J. Speech, 96, 115-140, https://doi.org/10.1080/00335631003796669, 2010.

Haubrich, D.: Sicher unsicher. Eine praktikentheoretische Perspektive auf die Un-/Sicherheiten der Mittelschicht in Brasilien, Bielefeld, 2015.

Henkel, R.: Are Geographers religiously unmusical? Positionalities in Geographical Research on Religion, Erdkunde, 65, 389-399, 2011.

Hillebrandt, F.: Soziologische Praxistheorien. Eine Einführung, Springer, Wiesbaden, 2014.

Joas, H.: Die Kreativität des Handelns, Suhrkamp, Frankfurt a. M., 1992. 
Lahr-Kurten, M.: Deutsch sprechen in Frankreich, Praktiken der Förderung der deutschen Sprache im französischen Bildungssystem, Bielefeld, 2012.

Latour, B.: Why Has Critique Run out of Steam? From Matters of Fact to Matters of Concern, Crit. Inquiry, 30, 225-248, 2004.

Latour, B.: Wir sind nie modern gewesen. Versuch einer symmetrischen Anthropologie, Suhrkamp, Frankfurt a. M., 2008.

Lorenz, E. N.: Predictability: does the flap of a butterfly's wings in Brazil set off a tornado in Texas? 139th Annual Meeting of the American Association for the Advancement of Science, 29 December 1972, in: Essence of Chaos, University of Washington Press, Seattle, Appendix 1, 181, 1995.

Löw, M.: Raumsoziologie, Suhrkamp, Frankfurt a. M., 2001.

MacKenzie, D., Muniesa, F. and Siu, L.: Do economists make markets? On the performativity of economics, Princeton University Press, Princeton, 2018.

Marston, S. A., Jones, J. P. und Woodward, K.: Human geography without scale, T. I. Brit. Geogr., 30, 416-432, 2005.

Massey, D.: Vocabularies of the economy, Soundings, 54, 9-22, 2012.

Maus, G.: Erinnerungslandschaften: Praktiken ortsbezogenen Erinnerns am Beispiel des Kalten Krieges, Kieler Geographische Schriften, 127, Geographisches Institut, Kiel, 2015.

Ouma, S. und Bläser, K.: Räume der Kalkulation, Kalkulation des Raumes. Geographien der finanziellen Ökonomisierung, Z. Wirtschaftsgeogr., 59, 214-229, 2015.

Ravix, J.-L.: Localization, Innovation and Entrepeneurship: An Appraisal of the Analytical Impact of Marshall's Notion of Industrial Atmosphere, J. Innovation Econ. Manage., 2, 63-81, 2012.

Sampson, T. D.: Virality. Contagion Theory in the Age of Networks, University of Minnesota Press, Minneapolis, London, 2012.

Schatzki, T. R.: Social Practices. A Wittgensteinian Approach to Human Activity and the Social, Cambridge University Press, Cambridge, 1996.
Schatzki, T. R.: The site of the social. A philosophical account of the constitution of social life and change, Pennsylvania State University Press, University Park, 2002.

Schatzki, T. R.: A New Societist Social Ontology, Philos. Soc. Sci., 33, 174-202, 2003.

Schatzki, T. R.: Social practices: a Wittgensteinian approach to human activity and the social, Cambridge University Press, Cambridge, 2008.

Schatzki, T. R.: The timespace of human activity: on performance, society, and history as indeterminate teleological events, Lexington Books, Lanham, 2010.

Schatzki, T. R.: Keeping Track of Large Phenomena, Geogr. Z., 104, 4-24, 2016.

Schäfer, S. und Everts, J.: Praktiken und Raum. Humangeographie nach dem Practice Turn, Transcript, Bielefeld, 2019.

Schmid, B.: Making Transformative Geographies. Lessons from Stuttgart's Community Economy, Transcript, Bielefeld, 2020.

Schmieder, J.: Mythos Garage, in: Süddeutsche Zeitung Online, abrufbar unter: https://www.sueddeutsche.de/wirtschaft/ urspruenge-von-apple-disney-barbie-und-harley-mythos-garage- 1. 1891487 (zuletzt abgerufen: 13. März 2020), 2014.

Silk, J.: Caring at a distance: gift theory, aid chains and social movements, Soc. Cult. Geogr., 5, 229-251, 2004.

Tapia, A. H.: Resistance or Deviance? A High-Tech Workplace During the Bursting of the Dot-Com Bubble, in: Information Systems Research, IFIP International Federation for Information Processing, edited by: Kaplan, B., Truex, D. P., Wastell, D., Wood-Harper, A. T. and DeGross, J. I., Vol. 143, Springer, Boston, 577-596, 2004.

Werlen, B.: Zur Ontologie von Gesellschaft und Raum. Sozialgeographie alltäglicher Regionalisierungen, Band 1, Franz Steiner Verlag, Stuttgart, 1999. 